\title{
Fluorescent Tagging of Rhythmically Active Respiratory Neurons within the Pre-Bötzinger Complex of Rat Medullary Slice Preparations
}

\author{
Silvia Pagliardini, ${ }^{\star}$ Tadafumi Adachi, ${ }^{\star}$ Jun Ren, Gregory D. Funk, and John J. Greer \\ Department of Physiology, Centre for Neuroscience, University of Alberta, Edmonton, Alberta, Canada T6G 2S2
}

\begin{abstract}
Elucidation of the neuronal mechanisms underlying respiratory rhythmogenesis is a major focal point in respiratory physiology. An area of the ventrolateral medulla, the pre-Bötzinger complex (preBötC), is a critical site. Attention is now focused on understanding the cellular and network properties within the preBötC that underlie this critical function. The inability to clearly identify key "rhythmgenerating" neurons within the heterogeneous population of preBötC neurons has been a significant limitation. Here we report an advancement allowing precise targeting of neurons expressing neurokinin-1 receptors (NK1Rs), which are hypothesized to be essential for respiratory rhythmogenesis. The internalization of tetramethylrhodamine conjugated substance $\mathrm{P}$ in rhythmically active medullary slice preparations provided clear visualization of NK1R-expressing neurons for subsequent whole-cell patch-clamp recordings. Among labeled neurons, $82 \%$ were inspiratory modulated, and $25 \%$ had pacemaker properties. We propose that this approach can be used to greatly expedite progress toward understanding the neuronal processes underlying the control of breathing.
\end{abstract}

Key words: breathing; apnea; medulla; neurokinin receptors; brainstem; substance $\mathrm{P}$

\section{Introduction}

The discovery of the pre-Bötzinger complex (preBötC) as a critical site for mammalian respiratory rhythmogenesis was a seminal advancement in the field of the neural control of mammalian breathing (Smith et al., 1991). The preBötC resides in a restricted region of the ventrolateral medulla at the rostral end of a column of neurons known for decades to be involved in respiratory control. The development of thin medullary slice preparations containing the preBötC provided a focal point for study and the promise of rapid progress toward final resolution of the mechanisms underlying rhythm generation. Indeed, this preparation has enabled application of varied fluorescent imaging techniques, made the whole-cell analysis of neurons in this region routine, and has contributed to significant advances in the field over the last decade (Smith et al., 1991; Frermann et al., 1999; Koshiya and Smith, 1999; Del Negro et al., 2002; Onimaru and Homma, 2003). Despite this, the fact that the preBötC contains a functionally heterogeneous pool of neurons has been a major remaining impediment to progress. It will be critical to specifically demarcate the target preBötC neurons of interest within viable, rhythmically active preparations. Here we present an important ad-

\footnotetext{
Received Dec. 3, 2004; revised Jan. 31, 2005; accepted Feb. 1, 2005.

This work was supported by the Canadian Institutes of Health Research (CIHR) and Alberta Lung Association. J.J.G. and G.D.F. are Scientist and Senior Scholar of the Alberta Heritage Foundation for Medical Research (AHFMR), respectively. S.P. and J.R. have been awarded studentships from AHFMR and CIHR.

*S.P. and T.A. contributed equally to this work.

Correspondence should be addressed to John J. Greer, Department of Physiology, Centre for Neuroscience, 513 Heritage Medical Research Centre, University of Alberta, Edmonton, Alberta, Canada T6G 2S2. E-mail: john.greer@ualberta.ca.

DOI:10.1523/JNEUROSCI.4930-04.2005

Copyright $\odot 2005$ Society for Neuroscience $\quad$ 0270-6474/05/252591-06\$15.00/0
}

vancement that provides for the rapid and precise targeting of this relatively small population of neurons. We exploited the fact that neurokinin-1 receptor (NK1R) expression identifies a subset of respiratory neurons within the preBötC that are hypothesized to play an important role in inspiratory rhythmogenesis (Gray et al., 1999, 2001; Pilowsky and Feldman, 2001; Guyenet et al., 2002; Manzke et al., 2003; Stornetta et al., 2003). Targeted destruction of these cells with substance P (SubP)-saporin completely disrupts breathing rhythm in vivo (Gray et al., 2001). We used fluorescently conjugated SubP, which is internalized after binding to NK1R receptors, in conjunction with fluorescent infrareddifferential interference contrast (IR-DIC) microscopy, to identify key neurons within the preBötC for subsequent whole-cell analysis. This approach markedly increases the probability of selecting precisely those neurons thought to be essential for mammalian respiratory rhythmogenesis.

\section{Materials and Methods}

Medullary slice preparation. Sprague Dawley rats were bred at the University of Alberta. The Animal Welfare Committee of the University of Alberta approved all procedures. Newborn rats (postnatal days 1-4) were used. Animals were anesthetized by inhalation of halothane or isofluorane (2-3\%), and the neuraxis was isolated. Rhythmically active medullary slice preparations were produced as described previously (Smith et al., 1991). Briefly, the brainstem-spinal cord was pinned, ventral surface upward, on a paraffin-coated metal plate. The plate was mounted in the vise of a Leica (Nussloch, Germany) VT1000S vibratome and sectioned serially. During visualization of appropriate landmarks, a single transverse slice containing the preBötC was cut $(550-650 \mu \mathrm{m}$ thick), transferred to a chamber containing artificial CSF (aCSF) (in mM: $128 \mathrm{NaCl}, 8.0 \mathrm{KCl}, 1.5 \mathrm{CaCl}_{2}, 1.0 \mathrm{MgSO}_{4}, 24 \mathrm{NaHCO}_{3}, 0.5 \mathrm{NaH}_{2} \mathrm{PO}_{4}$, 
and 30 D-glucose) equilibrated with $95 \%$ $\mathrm{O}_{2}-5 \% \mathrm{CO}_{2}$ at room temperature.

Tetramethylrhodamine conjugated-SubP application in living medullary slice. In experiments designed to assess the effects of tetramethylrhodamine-conjugated (TMR)SubP on rhythmic network activity and longterm viability, slices were placed in a "flowthrough" recording chamber $(2 \mathrm{ml})$ perfused with aCSF ( $2 \mathrm{ml} / \mathrm{min})$. TMR-SubP (500 nM; Molecular Probes, Eugene, OR) was administered to the perfusion solution for $5 \mathrm{~min}$. To assess the extent of TMR-SubP internalization or to label neurons for subsequent whole-cell analysis of respiratory neurons, slices were transferred to microcentrifuge tubes containing TMR-SubP (500 nM) in aCSF $(1 \mathrm{ml})$ and incubated in the dark for $5-8 \mathrm{~min}$ at $31^{\circ} \mathrm{C}$ under continuous delivery of $95 \% \mathrm{O}_{2}-5 \% \mathrm{CO}_{2}$. Slices were transferred to a recording chamber $(0.5 \mathrm{ml})$ perfused with aCSF $(2-3 \mathrm{ml} / \mathrm{min})$ for whole-cell analysis or fixed in $4 \%$ paraformaldehyde in phosphate buffer $(0.1 \mathrm{M}, \mathrm{pH}$ 7.2) for imaging. Our initial experiments were performed with a similar protocol but using the fluorochrome Oregon Green 488 conjugated with SubP (Molecular Probes).

Immunohistochemistry. Fixed medullary slices were processed for NK1R immunolabeling according to the following protocol. Slices were sectioned $(50 \mu \mathrm{m})$ on a vibratome. Sections were rinsed in PBS and incubated with $1.0 \%$ bovine serum albumin (BSA) (Sigma, St. Louis, $\mathrm{MO}$ ) and $0.3 \%$ Triton X-100 in PBS for 90 min to reduce nonspecific staining and increase antibody penetration. Sections were incubated overnight with primary antibody (1:1000; rabbit polyclonal anti-NK1R; Advanced Targeting Systems, San Diego, CA) diluted in PBS containing $0.1 \%$ BSA. After several washes in PBS, sections were incubated with cyanine 2 (Cy2)-conjugated donkey anti-rabbit (1:200; Jackson ImmunoResearch, West Grove, PA) diluted in PBS and 0.1\% BSA for $2 \mathrm{~h}$. Sections were further washed in PBS, mounted, and coverslipped with Fluorsave mounting medium (Calbiochem, La Jolla, CA). Alternatively, medullary slices were embedded in $6 \%$ agar, and $100-\mu \mathrm{m}$-thick coronal sections were cut with a vibratome to analyze the depth of TMR-SubP labeling in the tissue slice.

Confocal imaging of fixed tissue. Mounted sections were examined with a Zeiss (Oberkochen, Germany) 100M microscope ( $20 \times$ or $40 \times$ objective) using a LSM 510 NLO laser and LSM 510 software (Zeiss). For Cy2 fluorescence, excitation was set to $488 \mathrm{~nm}$, and emissions were collected with a $505 \mathrm{~nm}$ long-pass filter. For TMR-SubP fluorescence, excitation was set to $543 \mathrm{~nm}$, and emissions were collected using a $560 \mathrm{~nm}$ long-pass filter. Multiple, thin sections were acquired along the $z$-axis to assess extent of TMR-SubP labeling and the degree to which it colocalized with NK1R immunolabeling. Acquired images were exported in JPEG format, and brightness and contrast were adjusted in Adobe Photoshop 7.0 (Adobe Systems, Mountain View, CA).

Recording and analysis. Recordings of population inspiratory activity from hypoglossal (XII) nerve roots were made via suction electrodes. Signals were amplified, filtered $(0.3-3 \mathrm{kHz})$, rectified, integrated $(\tau=50$ $\mathrm{ms}$ ), and recorded on a computer using a Digidata 1322A analog-todigital board and Axoscope software (Axon Instruments, Union City, $\mathrm{CA})$. Whole-cell recordings were made from slices placed in a recording chamber on the fixed stage of an upright microscope (Zeiss Axioskop II FS) equipped with IR-DIC optics and epifluorescence. Slices were continuously perfused $(2-3 \mathrm{ml} / \mathrm{min})$ at $27^{\circ} \mathrm{C}$ with aCSF equilibrated with $95 \% \mathrm{O}_{2}-5 \% \mathrm{CO}_{2}$. A delay of at least $30 \mathrm{~min}$ was allowed before recording to allow for stabilization of respiratory rhythm and removal of nonspecific TMR-SubP fluorescence. TMR-SubP-positive neurons were identified under epifluorescence using a CCD camera (ICD-47; Ikegami, Tokyo, Japan) and monitor (M910; National Electronics), without any additional image intensification hardware or imaging software. We have since used a Dage-MTI (Michigan City, IN) IR-1000 camera that provides for yet a better detection of the fluorescent signal. Whole-cell re-

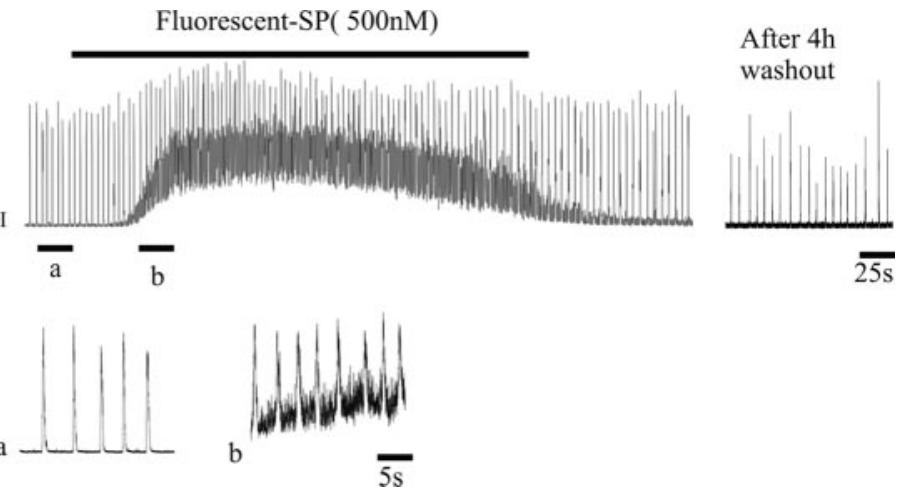

After $4 \mathrm{~h}$

$\overline{25 s}$

cordings were then obtained from TMR-SubP-positive neurons under IR-DIC visualization using patch electrodes (resistance, 3-4 M $\Omega$ ) pulled on a horizontal puller (model P-97; Sutter Instruments, Novato, CA) from $1.2 \mathrm{~mm}$ outer diameter filamented borosilicate glass (World Precision Instruments, Sarasota, FL). Fluorescent illumination was minimized by placing a blocking filter in the fluorescent light path as soon as a candidate TMR-SubP-positive neuron was identified. Pipettes were filled with potassium gluconate solution containing the following (in $\mathrm{mm}$ ): 122.5 potassium gluconate, $17.5 \mathrm{KCl}, 9 \mathrm{NaCl}, 1 \mathrm{MgCl}_{2}, 10 \mathrm{HEPES}$, 0.2 EGTA, 3 ATP-Mg, and 0.3 GTP-Tris, pH adjusted to 7.3 (with $5 \mathrm{~N}$ $\mathrm{KOH})$. Intracellular signals were amplified and filtered $(2-5 \mathrm{kHz}$ lowpass Bessel filter) with a multiclamp 700A amplifier (Axon Instruments) and acquired via a Digidata 1322A analog-to-digital board and pClamp 9.0 software. Series resistance and whole-cell capacitance were estimated under voltage-clamp conditions using short voltage pulses $(100 \mathrm{~Hz},-10$ $\mathrm{mV}, 3 \mathrm{~ms}$ ). Series resistance was compensated by $75 \%$ and monitored throughout the experiments. In cases in which permanent records of fluorescent and IR-DIC images of live neurons were acquired, we used a Zeiss Axiocam camera in conjunction with Axiovision software.

\section{Results}

\section{Diffusion and internalization of TMR-SubP in medullary slices}

To assess the diffusion of TMR-SubP and its effects on network activity, we applied it to the solution bathing medullary slice preparations. Similar to unconjugated SubP (Murakoshi et al., 1985; Yamamoto et al., 1992; Johnson et al.,1996), 500 nM TMRSubP elicited a markedly stimulated respiratory rhythm (Fig. 1). The potentiation was readily reversible during washout. Moreover, TMR-SubP did not affect preparation viability because the medullary slices generated a robust inspiratory output for at least $4 \mathrm{~h}$. These data indicate that the fluorescently tagged SubP diffuses into the medullary slice and binds to NK1R on neurons that regulate respiratory rhythm without having toxic effects.

SubP internalization within cells is thought to occur within minutes (for review, see Harrison and Geppetti, 2001; Mantyh, 2002). To assess the degree of internalization of TMR-SubP within the medullary slice preparation, we bathed slices in TMRSubP $(500 \mathrm{~nm})$ for $5 \mathrm{~min}$ at $31^{\circ} \mathrm{C}$. Slices were then fixed and sectioned in both the transverse and coronal planes, and the distribution of the fluorescent signal was assessed via confocal imaging. As shown in Figure 2, the distribution of fluorescent signal resulting from the internalization of TMR-SubP was similar to that achieved by immunolabeling for NK1R. Punctate immunofluorescence in both transverse and coronal sections was detected 


$$
\mathbf{a}
$$
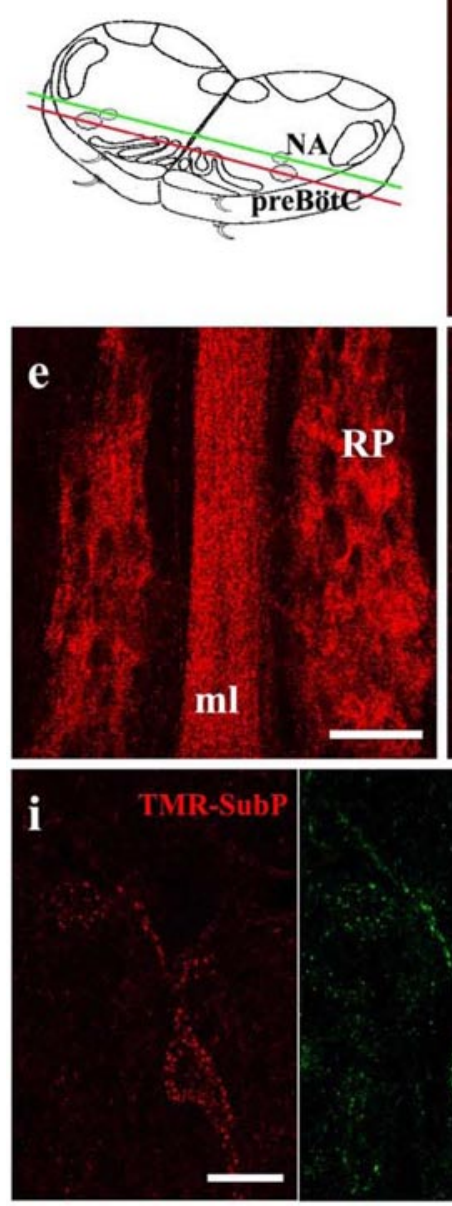
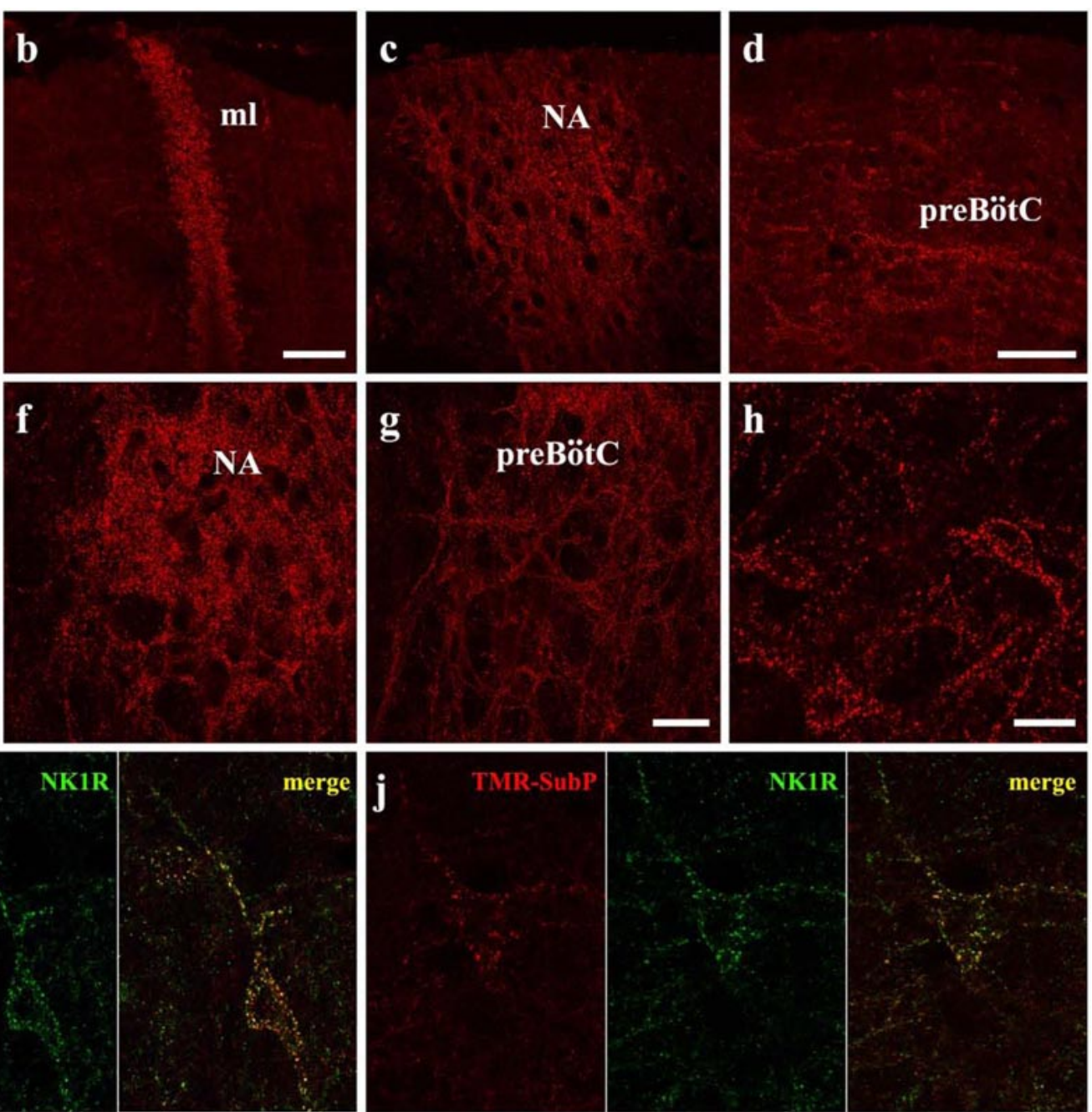

h
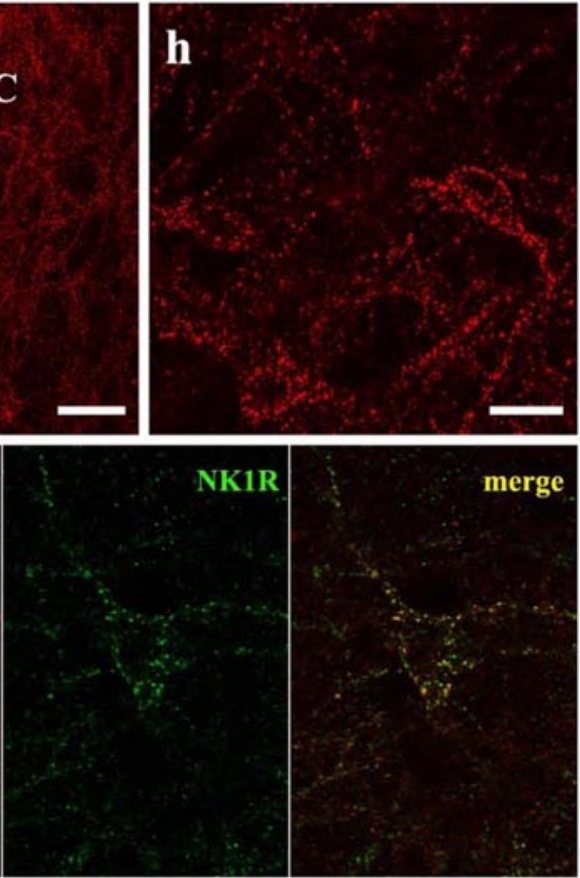

merge

Figure 2. Internalization of TMR-SubP in living medullary slice preparations. $\boldsymbol{a}$, Schematic drawing of medullary slices used for TMR-SubP internalization experiments. The green and red lines delineate the coronal plane from which images $\boldsymbol{b}$ and $\boldsymbol{c}$ (green) and $\boldsymbol{d}$ (red) were taken. $\boldsymbol{b}$ - $\boldsymbol{d}$, TMR-SubP internalization along the coronal plane at the midline (ml; $\boldsymbol{b}$ ), more laterally at the nucleus ambiguus (NA; $\boldsymbol{c}$ ), and more ventrolaterally at the preBötC (d). The top part of the figures corresponds to the rostral surface of the slice. $\boldsymbol{e}-\boldsymbol{h}$, TMR-SubP internalization in medullary slices along the transverse plane. A 5 min application of TMR-SubP (500 nm) results in robust internalization into neurons located in the midline, raphe pallidus (RP; $\boldsymbol{e})$, nucleus ambiguus ( $\boldsymbol{f})$, and preBötC $(\boldsymbol{g}, \boldsymbol{h})$. $\boldsymbol{i}$, $\boldsymbol{j}$, Colocalization of TMR-SubP fluorescence (red) and NK1R immunolabeling (green) in preBötC neurons. Fine and punctate staining for both NK1R and TMR-SubP is present in preBötC neurons. Note the high level of colocalization (yellow puncta) in the cell body and dendrites of preBötC neurons. Scale bars: (in $\boldsymbol{b}, \boldsymbol{d}, \boldsymbol{e}, \boldsymbol{g}), \boldsymbol{b}-\boldsymbol{g}, 50 \mu \mathrm{m} ;$ (in $\boldsymbol{h}, \boldsymbol{i}) \boldsymbol{h}-\boldsymbol{j}, 20 \mu \mathrm{m}$.

in cell bodies and dendrites within all regions of the slice that express NK1R during the perinatal period: midline (Fig. $2 b, e$ ), raphe nucleus (Fig. 2e), nucleus ambiguus (Fig. $2 c, f$ ), and preBötC (Fig. 2d,g,h) (Horie et al., 2000; Liu and Wong-Riley, 2002; Pagliardini et al., 2003). To further correlate the internalization of TMR-SubP with NK1R expression, treated slices were processed for immunohistochemical detection of NK1R. Double labeling, as indicated by fine yellow puncta, was observed in dendrites and cell bodies at all depths of the slice. This included strong colocalization within the preBötC (Fig. $2 i, j$ ). It appeared that all NK1R-immunopositive neurons had internalized TMR-SubP.

\section{Whole-cell recording from fluorescently tagged} preBötC neurons

We next combined the TMR-SubP labeling procedure with IRDIC optics to obtain whole-cell recordings of NK1R-expressing respiratory neurons within the preBötC of rhythmically active slice preparations. We targeted neurons located ventrolateral to the nucleus ambiguus in the rostral aspect of the medullary slices.
Within a single field of view, there were typically a few neurons on the surface that had intense, diffuse staining. As is often the case for surface cells of vibratome-prepared slices, these neurons showed evidence of fractured membranes and depolarized resting membrane potentials and were unable to fire action potentials. In neurons located two to three cell layers below the surface, labeling was punctate and outlined the surface of dendrites and somata. Targeting of these neurons for whole-cell patch recording under IR-DIC visualization provided a very high yield of recordings from healthy inspiratory neurons. In total, we recorded 34 TMR-SubP-positive preBötC neurons from 12 rhythmically active medullary slices (Fig. 3). Eighty-two percent (28 of 34) of neurons were rhythmically active inspiratory neurons with overshooting action potentials. The remaining six neurons fired robust trains of action potentials in response to depolarizing current but did not receive inspiratory-related synaptic drive potentials (data not shown). We then determined whether any of the fluorescently tagged preBötC neurons had characteristics consistent with pacemaker neurons, fitting with a prominent hypothesis that NK1R-positive neurons are important in rhythm gener- 
ation and, in turn, that pacemaker neurons are involved in respiratory rhythmogenesis (Rekling and Feldman, 1998; Smith et al., 2000). Putative pacemaker neurons within the preBötC discharged in-phase with inspiratory activity, but their oscillation frequency increases with progressive depolarization (i.e., they generate ectopic bursts). More definitive is that they continue to burst after disruption of network activity, for example after the removal of inspiratory drive transmission by blocking ionotropic, non-NMDA glutamate receptors (Johnson et al., 1994; Butera et al., 1999). We used the latter criteria after the observation that, in some neurons with voltage-dependent bursting properties, spontaneous burst firing stopped after disruption of network activity with CNQX. Of the 12 inspiratory neurons examined before and after blockade of network activity in $5 \mu \mathrm{M}$ CNQX, nine stopped firing, and all rhythmic synaptic activity was abolished after $\sim 15 \mathrm{~min}$. CNQX-sensitive neurons that were depolarized via injection of DC current did not burst but fired tonically. The remaining three $(25 \%)$ neurons continued to generate periodic bursting at a frequency and duration similar to the inspiratory-related motor output generated by the intact network in vitro (Fig. 3b, bottom recording). We did not systematically confirm under voltage-clamp conditions that all rhythmic synaptic activity was blocked by CNQX in these three neurons. However, the same concentration of CNQX has been used successfully in a similar preparation to synaptically isolate pacemaker neurons (Del Negro et al., 2002). The neuron shown in Figure $3 c$ demonstrated voltagedependent bursting in the presence of CNQX. Additional confirmation that the fluorescently tagged neurons were NK1R positive was achieved by applying the NK1R agonist, $\left[\operatorname{Sar}^{9}, \operatorname{Met}\left(\mathrm{O}_{2}\right)^{11}\right]$-SP (500 $\mathrm{nM})$ in the presence of TTX (400 nM). $\left[\mathrm{Sar}^{9}, \mathrm{Met}\left(\mathrm{O}_{2}\right)^{11}\right]$-SP produced a longlasting depolarization in all CNQXresistant neurons tested $(n=3)$ (Fig. $3 d)$.

\section{Discussion}

There is a concerted effort to elucidate the neuronal mechanisms operating within the preBötC because of its essential role for breathing in mammals. This includes an assessment of its ontogeny, cellular, network, and neuropharmacological properties. Advancement will depend critically on targeting of putative respiratory rhythmogenic neurons for intracellular recordings. Work in these areas has benefited greatly from the development of the brainstemspinal cord preparation by Suzue (1984) and the subsequent use of "blind" whole-cell recordings (Smith et al., 1988, 1992). Ac-
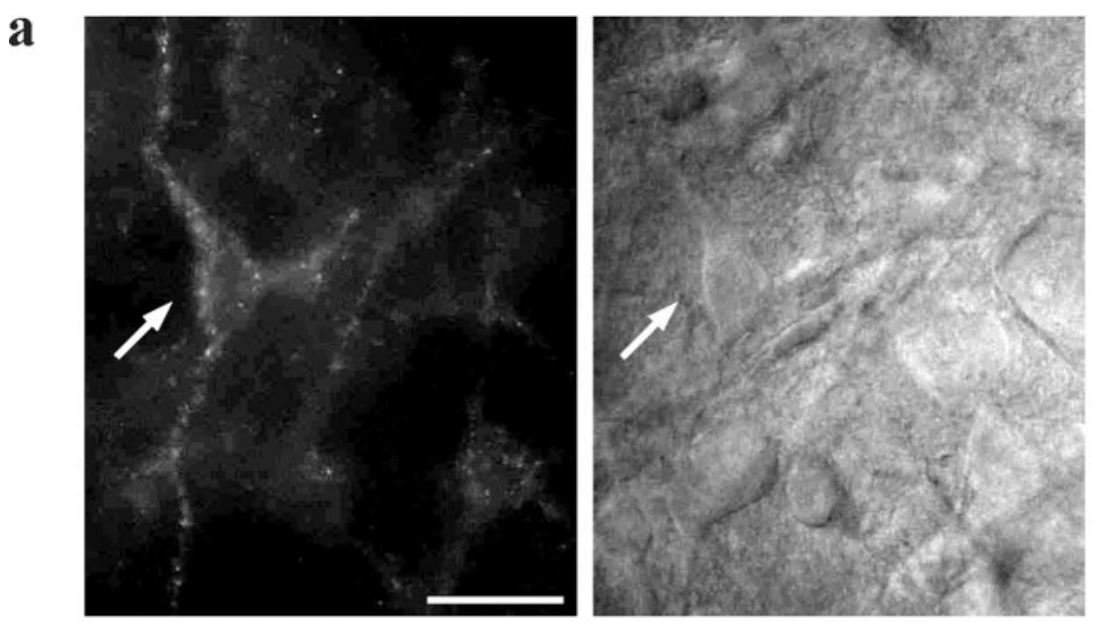

b Control

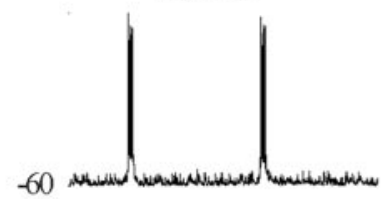

$\mathrm{CNQX}(5 \mu \mathrm{M})$

Washout
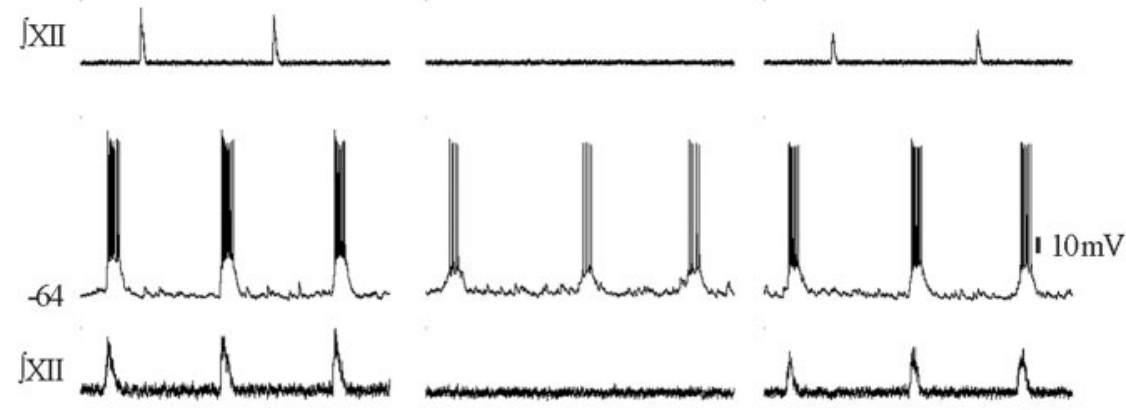

c

$\operatorname{CNQX}(5 \mu \mathrm{M})$

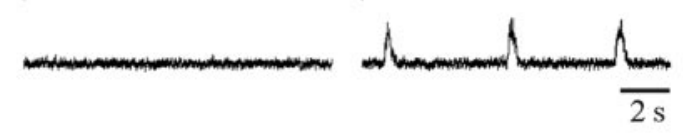

d
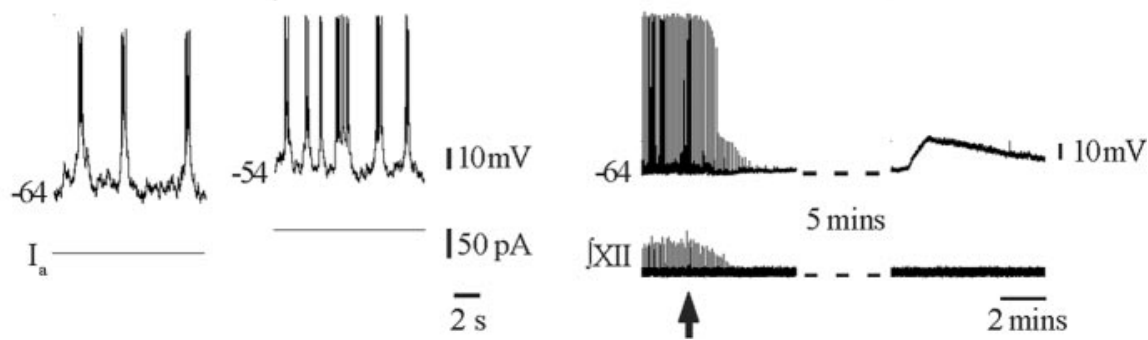

Figure 3. $\quad \boldsymbol{a}$, Fluorescent (left) and IR-DIC (right) image of a TMR-SubP-labeled preBötC neuron (arrow). Note that only a subpopulation of preBötC neurons visible under IR-DIC are TMR-SubP positive; $40 \times, 0.8$ numerical aperture Zeiss objective. Scale bar, $25 \mu \mathrm{m}$. $\boldsymbol{b}$, Whole-cell recording of membrane potential from two TMR-SubP-positive neurons and simultaneous recording of integrated XII nerve inspiratory activity before, during, and after block of rhythmic activity by bath application of CNQX. c, Whole-cell recording from a TMR-SubP-positive neuron that bursts in a voltage-dependent manner in response to 37 pA depolarizing bias current injection $\left(I_{\mathrm{a}}\right)$ after application of CNQX. $\boldsymbol{d}$, Whole-cell recording from a TMR-SubP fluorescent, CNQX-resistant, preBötC inspiratory neuron showing the membrane potential response evoked by local application of the NK1R agonist $\left[\right.$ Sar $\left.{ }^{9}, \operatorname{Met}\left(\mathrm{O}_{2}\right)^{11}\right]-S P(500 \mathrm{~nm})$ after block of rhythmic network activity by TTX (arrow indicates time of TTX application).

cessibility increased with the development of the medullary slice preparation, and whole-cell yields were enhanced with development of IR-DIC microscopy (Smith et al., 1991). Together, these techniques offered direct visualization and targeting of neurons within the ventrolateral medulla based on general morphological criteria. However, because of the functional heterogeneity of neu- 
rons within the region, which contains respiratory and nonrespiratory neurons, there were no reliable distinguishing criteria for targeting. Smith and colleagues developed the approach of retrogradely labeling propriobulbar neurons with calcium-sensitive dyes to allow for real-time dynamic fluorescent imaging of neuronal activity, including those with pacemaker properties, within the preBötC (Koshiya and Smith, 1999). More recently, it has become apparent that immunohistochemical labeling for NK1R and somatostatin in paraformaldehyde-fixed tissue can help delineate respiratory neurons within the preBötC that are thought to play a critical role in respiratory rhythmogenesis (Gray et al., 1999, 2001; Pilowsky and Feldman, 2001; Guyenet et al., 2002; Manzke et al., 2003; Stornetta et al., 2003). In this study, we provide a significant advancement by fluorescently tagging these neurons in viable, rhythmically active medullary slices.

SubP is a high-affinity ligand for the G-protein-coupled NK1R. During binding with NK1R, SubP recruits G-proteins to the receptor, which mediates an alteration of receptor distribution via internalization of the receptor-ligand complex, followed by recycling of the receptor into cell membrane (Wang and Marvizon, 2002; Roosterman et al., 2004).

The SubP-induced internalization and recycling of NK1R has been well characterized (Wang and Marvizon, 2002; Roosterman et al., 2004). Several fluorochrome compounds conjugated to SubP have been developed (Turcatti et al., 1997; Bennett and Simmons, 2001). Our initial labeling experiments used the fluorochrome Oregon Green 488 conjugated with SubP. Despite the high specificity of binding with NK1R, the signal was unstable and bleached rapidly in living slices visualized with standard epifluorescence. TMR-SubP proved much more effective, providing a more intense, stable fluorescent signal. Moreover, comparing the pattern of TMR-SubP labeling with immunohistochemical labeling for NK1R receptors indicated that the fluorescent tag specifically labeled the entire compliment of NK1R neurons contained within the boundaries of the rhythmic slice.

The number of NK1R neurons within each bilateral preBötC has been estimated to be as few as 300 (Gray et al., 2001). Thus, the chances of patching onto those neurons without a means of precise targeting are very restricted. Granted, there has been considerable success in routinely obtaining recordings of respiratory neurons using blind approaches. This has included recordings of neurons distributed within the ventrolateral medulla that demonstrate bursting properties in isolated preparations bathed in elevated extracellular potassium. However, the experimental approach described here allows one to target the specific population of NK1R-expressing respiratory neurons within the preBötC that are hypothesized to be directly involved in rhythmogenesis, i.e., the population of neurons whose selective destruction in vivo with SP-tagged saporin causes breathing to stop (Gray et al., 2001). NK1R-positive neurons could be readily visualized and recorded using a standard IR-DIC microscope equipped for electrophysiology and epifluorescence. Moreover, visualization was achieved with a standard IR camera and monitor and did not require elaborate image integration or processing. Data collected to date indicate that TMR-SubP-labeled cells within the preBötC are a functionally heterogeneous population of neurons, a high percentage of which are active during the inspiratory cycle. Furthermore, one-quarter of those characterized in detail had properties consistent with pacemaker neurons and thus provides the first definitive evidence that bursting pacemaker neurons in the preBötC have the NK1 receptor phenotype. The fluorescent tagging of neurons will also facilitate characterization of the molecular properties that define their ontogenic and functional prop- erties by allowing more precise neuronal targeting for single-cell reverse transcription-PCR or the collection of mRNA from multiple NK1R-expressing neurons using laser-capture technologies. It may also prove advantageous to combine TMR-SubP labeling with the use of voltage- and calcium-sensitive dyes to examine population-specific oscillatory behavior.

In summary, tagging of NK1R-expressing neurons within the preBötC via internalization of TMR-SubP will accelerate acquisition of intracellular data (via conventional and perforatedpatch whole-cell recording) required for defining cellular and synaptic properties of the preBötC and hasten progress toward understanding the processes underlying its role in the generation of respiratory rhythm.

\section{References}

Bennett VJ, Simmons MA (2001) Analysis of fluorescently labeled substance $\mathrm{P}$ analogs: binding, imaging and receptor activation. BMC Chem Biol 1:1-12.

Butera RJ, Rinzel J, Smith JC (1999) Models of respiratory rhythm generation in the pre-Bötzinger complex. I. Bursting pacemaker neurons. J Neurophysiol 82:382-397.

Del Negro CA, Morgado-Valle C, Feldman JL (2002) Respiratory rhythm: an emergent network property? Neuron 34:821-830.

Frermann D, Keller BU, Richter DW (1999) Calcium oscillations in rhythmically active respiratory neurones in the brainstem of the mouse. J Physiol (Lond) 515:119-131.

Gray PA, Rekling JC, Bocchiaro CM, Feldman JL (1999) Modulation of respiratory frequency by peptidergic input to rhythmogenic neurons in the pre-Bötzinger complex. Science 286:1566-1568.

Gray PA, Janczewski WA, Mellen N, McCrimmon DR, Feldman JL (2001) Normal breathing requires pre-Bötzinger complex neurokinin-1 receptor-expressing neurons. Nat Neurosci 4:927-930.

Guyenet PG, Sevigny CP, Weston MC, Stornetta RL (2002) Neurokinin-1 receptor-expressing cells of the ventral respiratory group are functionally heterogeneous and predominantly glutamatergic. J Neurosci 22:3806-3816.

Harrison S, Geppetti P (2001) Substance P. Int J Biochem Cell Biol 33:555-576.

Horie M, Miyashita T, Watabe K, Takeda Y, Kawamura K, Kawano H (2000) Immunohistochemical localization of substance $\mathrm{P}$ receptors in the midline glia of the developing rat medulla oblongata with special reference to the formation of raphe nuclei. Brain Res Dev Brain Res 121:197-207.

Johnson SM, Smith JC, Funk GD, Feldman JL (1994) Pacemaker behavior of respiratory neurons in medullary slices from neonatal rat. J Neurophysiol 72:2598-2608.

Johnson SM, Smith JC, Feldman JL (1996) Modulation of respiratory rhythm in vitro: role of $\mathrm{Gi} / \mathrm{o}$ protein-mediated mechanisms. J Appl Physiol 80:2120-2133.

Koshiya N, Smith JC (1999) Neuronal pacemaker for breathing visualized in vitro. Nature 400:360-363.

Liu Q, Wong-Riley MT (2002) Postnatal expression of neurotransmitters, receptors, and cytochrome oxidase in the rat pre-Botzinger complex. J Appl Physiol 92:923-934.

Mantyh PW (2002) Neurobiology of substance P and the NK1 receptor. J Clin Psychiatry 63 [Suppl 11]:6-10.

Manzke T, Guenther U, Ponimaskin EG, Haller M, Dutschmann M, Schwarzacher S, Richter DW (2003) 5-HT4(a) receptors avert opioid-induced breathing depression without loss of analgesia. Science 301:226-229.

Murakoshi T, Suzue T, Tamai S (1985) A pharmacological study on respiratory rhythm in the isolated brainstem-spinal cord preparation of the newborn rat. Br J Pharmacol 86:95-104.

Onimaru H, Homma I (2003) A novel functional neuron group for respiratory rhythm generation in the ventral medulla. J Neurosci 23:1478-1486.

Pagliardini S, Ren J, Greer JJ (2003) Ontogeny of the pre-Botzinger complex in perinatal rats. J Neurosci 23:9575-9584.

Pilowsky PM, Feldman JL (2001) Identifying neurons in the pre-Bötzinger complex that generate respiratory rhythm: visualizing the ghost in the machine. J Comp Neurol 434:125-127.

Rekling JC, Feldman JL (1998) Pre-Bötzinger complex and pacemaker neurons: hypothesized site and kernel for respiratory rhythm generation. Annu Rev Physiol 60:385-405. 
Roosterman D, Cottrell GS, Schmidlin F, Steinhoff M, Bunnett NW (2004) Recycling and resensitization of the neurokinin 1 receptor. Influence of agonist concentration and Rab GTPases. J Biol Chem 279:30670-30679.

Smith JC, Liu G, Feldman JL (1988) Intracellular recording from phrenic motoneurons receiving respiratory drive in vitro. Neurosci Lett 88:27-32.

Smith JC, Ellenberger HH, Ballanyi K, Richter DW, Feldman JL (1991) PreBötzinger complex: a brainstem region that may generate respiratory rhythm in mammals. Science 254:726-729.

Smith JC, Ballanyi K, Richter DW (1992) Whole-cell patch-clamp recordings from respiratory neurons in neonatal rat brainstem in vitro. Neurosci Lett 134:153-156.

Smith JC, Butera RJ, Koshiya N, Del Negro C, Wilson CG, Johnson SM (2000) Respiratory rhythm generation in neonatal and adult mammals: the hybrid pacemaker-network model. Respir Physiol 122:131-147.

Stornetta RL, Rosin DL, Wang H, Sevigny CP, Weston MC, Guyenet PG
(2003) A group of glutamatergic interneurons expressing high levels of both neurokinin-1 receptors and somatostatin identifies the region of the pre-Bötzinger complex. J Comp Neurol 455:499-512.

Suzue T (1984) Respiratory rhythm generation in the in vitro brain stemspinal cord preparation of the neonatal rat. J Physiol (Lond) 354:173-183.

Turcatti G, Zoffmann S, Lowe III JA, Drozda SE, Chassaing G, Schwartz TW, Chollet A (1997) Characterization of non-peptide antagonist and peptide agonist binding sites of the NK1 receptor with fluorescent ligands. J Biol Chem 272:21167-21175.

Wang X, Marvizon JC (2002) Time-course of the internalization and recycling of neurokinin 1 receptors in rat dorsal horn neurons. Brain Res 944:239-247.

Yamamoto Y, Onimaru H, Homma I (1992) Effect of substance P on respiratory rhythm and pre-inspiratory neurons in the ventrolateral structure of rostral medulla oblongata: an in vitro study. Brain Res 599:272-276. 